lumbar pain were reported in three cases. In the other case, it was a respiratory chart. The urinary and digestive signs were variable or absent in one case. Hyperleukocytosis with a positive CRP were constant. The cytobacterioligical urine exam practiced systematically was positive in 2 cases by isolating an Enterobacter Cloacoe in one case and an E Coli in another case, it revealed aseptic leukocyturia in one case and it showed no abnormality in the other case. The diagnosis of abscess was made thanks to the ultrasound-CT abdominal pairing performed in all patients. All patients were treated with intravenous antibiotic therapy. Percutaneous drainage was performed in one patient due to the inefficacy of antibiotic alone. The evolution was marked by apyrexia and improvement of other clinical and biological signs.

Conclusion The treatment of the kidney abscess is based on antibiotic therapy with or without percutaneous or surgical drainage depending on the size of the abscess and the clinical course.

\section{P661 EVALUATION OF CHILDHOOD URINARY TRACT INFECTION AND ANTIBIOTIC SUSCEPTIBILITY IN A TURKISH CENTER}

${ }^{1}$ Ipek Kaplan Bulut, ${ }^{2}$ Alper Tunger, 'Sevgin Taner, ${ }^{3}$ Ezgi Boluk, ${ }^{1}$ Ahmet Keskinoglu, ${ }^{1}$ Caner Kabasakal ${ }^{*}$, ${ }^{2}$ Sohret Aydemir. ${ }^{1}$ Ege University Faculty of Medicine, Pediatric Nephrology, Izmir, Turkey; ${ }^{2}$ Ege University Faculty of Medicine, Medical Microbiology, Izmir, Turkey; ${ }^{3}$ Ege University Faculty of Medicine, Pediatrics, Izmir, Turkey

10.1136/archdischild-2019-epa.991

Introduction Urinary tract infection (UTI) is the most common bacterial infection in children under 2 years of age. Proper and timely treatment protects patients from long-term complications such as renal scarring, hypertension, and end-stage renal disease.

Methods The file records of 729 patients aged between 0-18 years, followed up with diagnosis of urinary tract infection, vesicoureteral reflux and neurogenic bladder in Ege University Pediatric Nephrology Unit between February 2013 and November 2018 were retrospectively reviewed.

Results A total of 1126 positive urine cultures of 729 patients $(65 \%$ female $/ 35 \%$ male) were included in the study. The mean infection time was $56 \pm 53$ months. Most of the cultures $(88.2 \%)$ were gram negative. Escherichia coli (E. coli) was the most common isolated bacteria with a prevalence of $59.1 \% \quad(\mathrm{n}=666)$ followed by Klebsiella pneumonia with 17.9\% ( $\mathrm{n}=202)$, Enterococcus faecalis $8.3 \%(\mathrm{n}=93)$, Proteus mirabilis 3.2\% $(\mathrm{n}=37)$, Enterococcus faecium $2.9 \%(\mathrm{n}=33)$ and Pseudomonas aeruginosa $2.5 \%(\mathrm{n}=28)$. Enterobacteriaceae in 962 cultures $(85.4 \%)$ constitute most bacteria in this study. Ampicillin, cefuroxime and trimethoprim-sulfamethoxazole with susceptibility rates of $18.6 \%, 39.6 \%, 49.0 \%$ respectively, constitute of the highest resistant antimicrobials to Enterobacteriaceae. Antimicrobials with the highest susceptibility in this group were meropenem, imipenem and ertapenem with susceptibility rates of $99.2 \%, 97.1 \%$ and $96.1 \%$ respectively. Enterococcus spp. showed the highest resistance to gentamycin with 50\% resistance to tested cases. Pseudomonas spp. with $64.3 \%$ susceptibility, showed the highest resistance to piperacillin-tazobactam.

Conclusion Enteric bacteria, majority of which constituted by E.Coli are at the forefront in the etiology of UTI. Diagnosis and accurate treatment of the disease prevents or reduces late complications and morbidity. Determination of local antibiotic susceptibility patterns, that expected differ by region and time, has a great importance for initiating effective empirical treatment and prevent complications.

\section{P662 DOES ULTRASOUND MEASURED RENAL LENGTH AND VOLUME DISCREPANCY PREDICT DIFFERENTIAL FUNCTION ON DMSA SCANNING?}

${ }^{1}$ Ciannait Lehane*, ${ }^{2}$ Stephanie Ryan, ${ }^{2}$ Gabrielle Colleran. ${ }^{1}$ Department of Paediatrics, Children's Health Ireland at Temple Street, Dublin, Ireland; 'Department of Radiology, Children's Health Ireland at Temple Street, Dublin, Ireland

\subsection{6/archdischild-2019-epa.992}

Investigation of children with urinary tract infection (UTI) or other renal disease ofteninvolvesa combination of dimercaptosuccinic acid (DMSA) imaging and ultrasound (US) imaging to identify structural and functional defects.

DMSA imaging provides information on the relative function of the right and left kidneys and is considered the goldstandard imaging modality for the detection of renal scarring. DMSA imaging requires anintravenous injection of a radioactive agent and often requires sedationin babies and young children. DMSA imaging takes more than two hours to complete and is not suitable for frequent repeat studies.

US imaging provides information on renal size, parenchymal appearance and structural abnormalities such as calyceal and/or renal pelvic dilatation. US imaging is non-invasive, does not involve ionising radiation and can be performed in most children without sedation or anaesthetic. US imaging takes less than 30 minutes to complete and can be repeated easily for follow up.

We have obtained ethical approval to investigate the associations between DMSA-assessed renal function discrepancy with US assessment of renal length, renal volume, renal length discrepancy and renal volume discrepancy. Image data will be captured and analysed from children who underwent both DMSA imaging and renal US imaging in our hospital between 2015 and 2018. The analytical group $(n=200)$ has been limited to those who underwent imaging as part of investigation of UTI or other renal disease who had DMSA and ultrasound scanning within six months of each other and whose images are adequate to obtain the relevant measurements.

Finding strong associations and sufficient diagnostic accuracy may mean that the non-invasive and more easily performed US examination, which does not involve ionising radiation, may reduce the need for DMSA scanning in some children. 